\title{
Cardiac Vagal Tone and Anthropometric Parameters in Healthy Young Adults- Can Altered Cardiac Vagal Tone Predict Development of Obesity? \\ Authors
}

\author{
Neetha Shastry ${ }^{1}$, Ashwini $^{2}$, Namratha Shetty ${ }^{3}$, Amrit M. Mirajkar ${ }^{4}$ \\ ${ }^{1,2}$ Assistant Professor, Department of Physiology, K S Hegde Medical Academy, Nitte University, \\ Deralakatte, Mangalore, Karnataka \\ ${ }^{3}$ Post Graduate Student, Department of Physiology, K S Hegde Medical Academy, Nitte University, \\ Deralakatte, Mangalore, Karnataka \\ ${ }^{4}$ Professor, Department of Physiology, K S Hegde Medical Academy, Nitte University, Deralakatte, \\ Mangalore, Karnataka \\ Corresponding Author
}

\section{Dr Ashwini}

Assistant Professor, Department of Physiology, K S Hegde Medical Academy, Mangalore, Karnataka, India Phone [or Mobile] No: +91 9740531995, Email: prasadashwini2@gmail.com

\section{ABSTRACT}

Background: This study was conducted to evaluate the effect of Cardiac vagal tone (CVT) on Body Mass Index (BMI) and Body fat percentage BFP) in healthy young adults.

Methodology: The study was performed on 121(males=59, female=62) healthy volunteers. The weight $\mathrm{kg}$ ) and heightm) were measured and BMI was calculated. BFP was calculated using Durenberg equation. Cardiac vagal measures deep breathing difference DBD) and Valsalva ratio VR) were evaluated. BMI, BFP, $D B D$ and VR were compared between both genders. The correlation between the anthropometric measures and CVT was determined by Pearson's/Spearman's correlation test. The effect of CVT on BMI and BFP was assessed by regression analysis.

Results: BMI was lower in females, whereas BFP was lower in males. No significant difference was found in the measures of cardiac vagal activity between the genders. A significant negative correlation was found between BMI and $D B D(r=-0.367) ;$ Body fat percentage and $D B D(r=-0.330)$ in males. Also, a significant negative correlation was found between cardiac VR and BMI $r=-0.317) ; V R$ and BFP $(r=-0.290)$ in males.No such correlation was found in females. Regression analysis showed CVT predicts BMI and BFP in males.

Conclusion: The present study concluded that BMI and BFP are associated with CVT in healthy young males and CVT predicts the development of obesity in healthy young males.

Keywords-Body Mass Index, Body Fat Percentage, Cardiac Vagal tone.

\section{INTRODUCTION}

Changes in lifestyle, dietary patterns and various environmental factors, genetic factors have resulted in increased prevalence of obesity ${ }^{[1]}$.

Overweight and obesity are the leading risk factors for development of cardiovascular disease (CVD). It accounts for $23 \%$ of the ischemic heart disease burden $^{[2]}$. Large prospective studies such as the Framingham Heart Study, have shown that overweight and obesity are associated with increased risk of CVD ${ }^{[3]}$. Obesity is known to be associated with increased risk of diabetes, hypertension, dyslipidemia and coronary artery disease ${ }^{[4]}$. Prevalence of obesity among young 
adults in developing countries range from 2.3 to 12 $\%$, with over weight as high as $28 \%{ }^{[5]}$. Increasing trend of obesity in young adults has been noted in last decade.

Obesity develops due to long term imbalance between energy intake and energy expenditure. Several factors like physical activity level, hormones and autonomic nervous system (ANS) play important role in the pathophysiology of obesity through regulation of energy expenditure ${ }^{[6]}$. The relationship between ANS and obesity is complex. Studies have shown association between low sympathetic activity and development of obesity ${ }^{[7,8]}$. It is also observed that obesity alters the activity of ANS. A $10 \%$ increase in body weight above an individual's usual weight causes a decrease in parasympathetic tone and increases the sympathetic activity ${ }^{[9]}$.

Body Mass Index (BMI) has been used as surrogate marker of obesity. But Body fat percentage (BFP) is considered to be a more reliable marker, as BMI cannot differentiate between fat mass and muscle mass. Cardiovagal domain of ANS can be assessed by evaluating heart rate responses during deep breathing manoeuvre and Valsalva manoeuvre ${ }^{[10]}$. Deep breathing test evaluates the parasympathetic functions whereas Valsalva manoeuvre evaluates the CVT using heart rate responses ${ }^{[11]}$.

Only few studies have evaluated the effects of alterations in parasymapathetic limb of ANS on body weight. Existing studies support the fact that cardiac vagal tone(CVT) varies inversely with markers of obesity, research studies of CVT in healthy young non-obese individuals (BMI $<30 \mathrm{~kg} / \mathrm{m} 2$ ) and its effect on BMI and BFP are sparse. We hypothesized that, a reduced CVT may anticipate the development of obesity in otherwise non-obese healthy adults. This study aimed to analyse the effect of CVT on BMI and BFP and to evaluate its role in gender specific prediction of development of obesity in young adults.

\section{MATERIALS AND METHODS}

This was a cross-sectional study involving 121(59 males, 62 females) healthy adults between the ages of 18 and 25 years. Subjects with acute illness in preceding two weeks, Diabetes mellitus, hypertension or other medical illness requiring treatment habitual use of tobacco and alcohol were excluded. Volunteers were requested to refrain from strenuous physical activity for 24hours, and any food or beverages for 2 hours prior to recording. Female volunteers performed experimental protocol between the 5 th to 10 th day of menstrual cycle to avoid confounding factors related to hormonal factors. Ethical clearance was obtained from Institutional Ethics and Research Committee and all the tests were performed after obtaining written informed consent from volunteers. The anthropometric parameters like weight $[\mathrm{kg}]$, and height $[\mathrm{m}]$ were measured using digital weighing scale, and stadiometer, respectively. The BMI was calculated from weight divided by height squared. Body fat percentage was calculated using Durenberg equation ${ }^{[12]}$. Following 15 minutes of rest, tests to assess cardiac parasympathetic function were performed. Resting heart rate was recorded using R-R interval of Lead II ECG. Resting baseline blood pressure was recorded. Simple tests based on the measurement of ECG derived heart rate responses to physiological perturbations of cardiovascular function were performed. These includes: Heart rate response to deep breathing Deep Breathing Difference (DBD) and Heart rate response to Valsalva Manoeuvre - Valsalva ratio (VR). These tests were performed using Labscribe software.

Heart rate responses to deep breathing manoeuvre was performed by connecting ECG electrodes for recording lead II ECG and respiratory belt transducer for recording chest movements. After obtaining, baseline recording for 30 seconds, deep breathing manoeuvre was performed.

Subject was instructed to breathe (inhalation and exhalation) at a slow and steady rate taking about 5 seconds each for inhalation and exhalation for at least six complete respiratory cycles. The ECG, thus obtained was analysed for maximum (HR max) and minimum heart rate (HR min) for each respiratory cycle, for six respiratory cycles. Difference between HR max and HR min was calculated for each cycle 
and mean of this difference was noted as DBD. A value $>16$ was considered to be normal.

Following 30 minutes of rest, heart rate responses to Valsalva manoeuvre was performed by asking the subject to blow into the mouthpiece (flange between the teeth, lips enclosing mouth piece, nose closed by nose clip), attached to the pressure transducer. After initial 30 seconds of recording in resting state, the subject was instructed to blow into mouthpiece with an open glottis, such that the intrathoracic pressure rises rapidly to $40 \mathrm{mmHg}$ and remains steady at that level for at least 15 seconds. After 15 seconds of steady pressure at $40 \mathrm{mmHg}$, subject was asked to release the pressure and continue breathing naturally. Recording was continued for 45 seconds after release of pressure. The maximum heart rate during the manoeuvre and minimum heart rate following the manoeuvre was noted. The ratio between the same was calculated. Valsalva Ratio $>1.45$ was considered to be normal.

Statistical analysis: The data obtained were analysed using SPSS version 19.0 software. Data was expressed as Mean \pm SD. Association between anthropometric parameters, vagal tone parameters among either gender was performed using independent $\mathrm{t}$ test. All vagal tone parameters were normally distributed except DBD in females and VR in both genders. After log transformation DBD normally distributed. Therefore parametric tests were used to analyse data of females. However, in males, VR remained same even after log transformation, hence; Spearman's correlation test was used. Regression analysis was performed to predict the relation of changes in parasympathetic tone and cardiovascular risk. $\mathrm{P}<0.05$ was taken as statistically significant.

\section{RESULTS}

Data from 121(59 males, 62 females) was utilised for analyses. Mean age of the study population was $19.18 \pm 1.15$ years.

Table 1 shows anthropometric parameters and cardiovascular parameters among either gender. There was significant difference in height, weight, BMI and BFP between males and females. Height, weight and BMI were significantly lower in females. However, BFP was significantly higher in females when compared with males. There was no significant difference in resting cardiovascular parameters between males and females. Difference among vagal tone parameters i.e. Deep breathing difference (DBD) and valsalva ratio (VR) among males and females was not found to be significant.

Table 1: Anthropometric Parameters and Cardiovascular Parameters

\begin{tabular}{l|c|c}
\hline Variables & \multicolumn{2}{|c}{ Mean \pm S.D } \\
\hline Anthropometric Parameters & Male[n=59] & Female[n=62] \\
\hline \hline Age(years) & $19.39 \pm 1.86$ & $18.97 \pm 1.14$ \\
\hline Height(m)* & $1.70 \pm 0.93$ & $1.60 \pm 0.06$ \\
\hline Weight(kg)* & $66.59 \pm 12.64$ & $53.44 \pm 7.97$ \\
\hline BMI(kg/m2)* & $23.05 \pm 3.39$ & $20.81 \pm 2.68$ \\
\hline Body fat percentage* & $15.96 \pm 4.01$ & $23.79 \pm 3.23$ \\
\hline Cardiovascular Parameters & & \\
\hline \hline Resting Heart rate [beats per minute] & $69.36 \pm 11.23$ & $74.48 \pm 13.82$ \\
\hline Resting SBP[mmHg] & $114.67 \pm 8.36$ & $112.07 \pm 7.59$ \\
\hline Resting DBP[mmHg] & $67.67 \pm 6.15$ & $65.27 \pm 4.50$ \\
\hline DBD & $29.32 \pm 10.80$ & $28.83 \pm 11.95$ \\
\hline VR & $1.81 \pm 3.30$ & $1.74 \pm 0.32$ \\
\hline
\end{tabular}

BMI: Body mass index, SBP: Systolic blood pressure, DBP: Diastolic blood pressure, DBD: Deep Breathing Difference, VR: Valsalva Ratio *Significant pvalue $<0.05$

Table 2 shows correlation between cardiac vagal measures and anthropometric parameters among males and females. There was significant negative correlation between DBD and Fat percentage in males Also, there was significant negative correlation between DBD and BMI in males. A negative correlation was found between VR and body fat percentage, BMI in males. There was no significant correlation between vagal tone parameters and anthropometric parameters among females.

Regression analysis was carried out to assess whether vagal tone as assessed by deep breathing and VR contributes to BMI and BFP in males (Table 3). DBD was found to have significant impact on BMI and BFP in males. Cardiac vagal measure VR was also found to statistically significantly predict BMI and BFP. 
Table 2: Correlation between cardiac vagal measures and anthropometric parameters

\begin{tabular}{l|l|l|l|l}
\hline & \multicolumn{2}{|l|}{ DBD } & VR \\
\cline { 2 - 5 } & $\begin{array}{l}\text { Male } \\
\text { r-value }\end{array}$ & $\begin{array}{l}\text { Female } \\
\text { r-value }\end{array}$ & $\begin{array}{l}\text { Male } \\
\text { r-value }\end{array}$ & $\begin{array}{l}\text { Female } \\
\text { r-value }\end{array}$ \\
\hline BMI & $-0.377 *$ & 0.748 & $-0.317^{*}$ & -0.142 \\
\hline BFP & $-0.339^{*}$ & 0.723 & $-0.290^{*}$ & -0.142 \\
\hline \hline *Significant p-value <0.05
\end{tabular}

As no significant correlation was found between the markers of CVT and anthropometeric measures in females, regression analysis was not carried out.

Table 3: Regression analysis of BMI \& BFP ( dependable variables) with DBD and VR 9independent variables) in males

\begin{tabular}{l|c|c|c}
\hline & $\begin{array}{c}\text { Standardised regression } \\
\text { Variables }\end{array}$ & t-value & P-value \\
\hline \hline $\begin{array}{l}\text { Regression analysis of BMI and BFP with DBD in males } \\
\text { BMI }\end{array}$ & -0.377 & -3.076 & 0.003 \\
\hline BFP & -0.339 & -2.272 & 0.009 \\
\hline \hline Regression analysis of BMI and BFP with VR in males & -2.163 & 0.008 \\
BMI & -0.317 & -2.457 & 0.004 \\
\hline BFP & -0.290 & Breathing Difference, \\
\hline BMI: Body Mass Index, BFP: Body Fat Percentage, DBD:Deep & \\
VR:Valsalva Ratio. \\
p-value < 0.05 significant
\end{tabular}

\section{DISCUSSION}

This is a cross sectional study among healthy volunteers of either gender. The results of present study suggested that in apparently healthy nonobese males the CVT is inversely associate with BMI and BFP. In present study the baseline characteristics showed that, height, weight and BMI was significantly higher in males. A cross sectional study that mainly assessed the association between anthropometric parameters and cardiovascular risk among young adults showed that, height, weight, waist circumference, hip circumference were found to be higher in males as compared to females ${ }^{[13]}$. Similar findings were found by a study, which showed that, the trend of overweight and obesity was more among males ${ }^{[14]}$. Although BMI was more in males, the BFP estimated by any standard technique is more among females when compared with males ${ }^{[15]}$. These findings are in line with the results of our study. Lower BFP among males may be attributed to greater lean body mass in them, that results in the greater BMI ${ }^{[16]}$.

It is well recognized that, cardiovascular responses of blood pressure, cardiac output, heart rate, and other variables to change in posture, breathing manoeuvres differ between genders. Several studies have found a significant difference in the cardiac autonomic activity between males and females of younger population ${ }^{[17,18]}$. Several studies on gender based differences in resting CVT have provided conflicting results. Some studies have shown increased cardiac vagal modulation in females ${ }^{[17-19]}$ while some have showed no such differences ${ }^{[20,21]}$. In the present study no significant difference was found in CVT between males and females. It is well established fact that young women are at lesser risk of developing hypertension and coronary artery disease than men of same age. This is attributed to the differences in cardiac autonomic modulations between the genders. Though in present study cardiac sympathetic modulation was not assessed, a recent study found no gender differences in cardiac vagal parameters in healthy volunteers, whereas it reported higher sympathetic activity in males compared to females ${ }^{[22]}$. The higher sympathetic tone in males can be attributed to the higher incidence of hypertension and coronary heart diseases in men.

In present study a significant negative correlation was found between BMI and CVT as assessed by DBD and VR in males. Also, a negative significant correlation was found between BFP and measures 
of CVT (Table 2). These findings suggest an association between CVT and anthropometric parameters in healthy young non-obese males. Previously, studies have evaluated the effect of BMI on vagal tone. A study assessed the influence of gender and BMI on vagal tone among adult population of mean age 38 years. It concluded that, among males, BMI had significant negative correlation with parasympathetic activity than females of same age group ${ }^{[23]}$. Our study findings are in agreement with the studies that have found a lower vagal tone in obese individuals ${ }^{[24]}$. Present study showed no correlation between cardiac vagal tone and anthropometric measures in females.

The outcomes of present study, give impression that decreased CVT plays a vital role in the shift of anthropometric measures from normal to overweight state in young males. Furthermore, measures of CVT emerged as significant contributor to BMI and BFP in young healthy males(Table3). The findings of this study suggest that, even in healthy young men, it is possible to identify the individuals with higher tendency to develop obesity by assessing CVT using simple tests like DBD and $\mathrm{VR}$ and take appropriate preventive measures.

\section{CONCLUSION}

This study demonstrated that cardiac vagal tone is associated with BMI and Body fat percentage in healthy young males and cardiac vagal tone predicts BMI and body fat percentage in healthy young males.

\section{AKNOWLEDGEMENTS}

The investigators, staff and participants of the study are gratefully acknowledged.

\section{Declarations: None}

Funding: None

Conflict of interest: None

\section{REFERENCES}

1. Mellati AA, Mousavinasab SN, Sokhanvar S, Kazemi SAN, Esmailli MH, Dinmohamadi H. Correlation of anthropometric indices with common cardiovascular risk factors in an urban adult population of Iran: data from Zanjan Healthy Heart Study. Asia Pac J ClinNutr. 2009;18(2):217-25.

2. Moran AE, Forouzanfar MH, Roth GA, Mensah GA, Ezzati M, Flaxman A, et al. The Global Burden of Ischemic Heart Disease in 1990 and 2010 The Global Burden of Disease 2010 Study. Circulation. 2014 Apr 8;129(14):1493-501.

3. Goh LGH, Dhaliwal SS, Welborn TA, Lee AH, Della PR. Anthropometric measurements of general and central obesity and the prediction of cardiovascular disease risk in women: a cross-sectional study. BMJ Open. 2014 Feb 1;4(2):e004138.

4. Kataria SK, Srivastava Dadhich A. The study of anthropometric parameters to study cardiovascular disease risk factors in adult population of westren Rajasthan. J AnatSoc India. 2010;59(2):211-5.

5. AmudhaPoobalan, Lorna Aucott. Obesity Among Young Adults in Developing Countries: A Systematic Overview. Current Obesity Reports. 2016; 5: 2-13.

6. Tataranni PA. From physiology to neuroendocrinology: a reappraisal of risk factors of body weight gain in humans. Diabetes Metab. 1998 Apr;24(2):108-15.

7. Tataranni PA, Young JB, Bogardus C, Ravussin E. A low sympathoadrenal activity is associated with body weight gain and development of central adiposity in Pima Indian men. Obes Res. 1997 Jul;5(4):341-7

8. van Baak, M. A. (2001) The peripheral sympathetic nervous system in human obesity. Obes Rev. 2: 3-14.

9. Young JB, Macdonald IA. Sympathoadrenal activity in human obesity: heterogeneity of finding since 1980. Int J Obes Relat Metab Disord. 1992;16:959-967.

10. Novak P. Quantitative autonomic testing. J Vis Exp. 2011 Jul 19;(53). pii: 2502. doi: $10.3791 / 2502$. 
11. Jaradeh SS, Prieto TE. Evaluation of the autonomic nervous system. Phys Med RehabilClin N Am. 2003 May;14(2):287305

12. Deurenberg P, Weststrate JA, Seidell JC. Body mass index as a measure of body fatness: age- and sex-specific prediction formulas.Br J Nutr. 1991 Mar;65(2):105-14

13. Kishan A. A Cross sectional study Oof anthropometric, blood pressure parameters and metabolic profile among medical students- gender differenece determines cardiovascular risk. IJPBCS. 2016;5(2):110.

14. Kuan PX, Ho HL, Shuhaili MS, Siti AA, Gudum HR. Gender differences in body mass index, body weight perception and weight loss strategies among undergraduates in Universiti Malaysia Sarawak. Malays J Nutr. 2011 Apr;17(1):67-75.

15. Gruen DG, Connor EB. Sex Differences in Measures of Body Fat and Body Fat Distribution in the Elderly. Am J Epidemiol. 1996;143(9):898-907.

16. Ley CJ, Lees B, Stevenson JC. Sex- and menopause-associ- ated changes in bodyfat distribution. Am J ClinNutr. 1992;55: 950-4.

17. ShailajaMoodithaya, Sandhya T. Avadhany.Gender Differences in AgeRelated Changes in Cardiac Autonomic Nervous Function. Journal of Aging Research Volume 2012 (2012), Article ID 679345, 7 pages

18. K. Umetani, D. H. Singer, R. McCraty, and M. Atkinson, "Twenty-four hour time domain heart rate variability and heart rate: relations to age and gender over nine decades," Journal of the American College of Cardiology, vol. 31, no. 3, pp. 593-601, 1998.

19. Heikki V. Huikuri, Sirkku M. Pikkuja“msa“, K.E. Juhani Airaksinen, Markku J. Ika“heimo, Asko O. Rantala, Heikki Kauma, MaunoLilja, Y. Antero Kesa“niemi. Sex- Related Differences in Autonomic
Modulation of Heart Rate in Middle-aged Subjects. Circulation. 1996;94:122-125

20. Niewiadomski W, Gasiorowska A, Krauss B, Mróz A, Cybulski G. Suppression of heart rate variability after supramaximal exertion. Clin Physiol Funct Imaging. 2007 Sep;27(5):309-19

21. Joyce M. Evans, Michael G. Ziegler, Abhijit R. Patwardhan, J. Blaine Ott, Charles S. Kim, Fabio M. Leonelli, Charles F. Knapp. Gender differences in autonomic cardiovascular regulation: spectral, hormonal, and hemodynamic indexes. Journal of Applied Physiology Published 1 December 2001 Vol. 91 no. 6, 2611-2618

22. D. Ramaekers, H. Ector, A. E. Aubert, A. Rubens and F. Van de Werf. Heart rate variability and heart rate in healthy volunteers. European Heart Journal (1998) 19, 1334-1341

23. Nayak. Influence of gender and body mass index on autonomic reactivity in adults and middle-aged population. International Journal of Clinical and Experimental Physiology. 2014: 1(4):3030-306.

24. Laederach-Hofmann K, Mussgay L, Ruddel H. Autonomic cardiovascular regulation in obesity. J Endocrinol. 2000;164:59-66. 